\title{
Desarrollo de los Islotes de Langerhans en Páncreas de Alpacas Neonatas
}

\author{
Development of Islets of Langerhans in Pancreas of Neonatal Alpacas \\ José Rodríguez G. ${ }^{1,5}$, Juan Espinoza B. ${ }^{2}$, Gabriela Rojas Z. ${ }^{3}$, Boris Lira M. ${ }^{3}$, \\ María Vásquez C. ${ }^{3}$, Bernardo López-Torres ${ }^{2}$, César Caro M. ${ }^{4}$
}

\section{Resumen}

El objetivo del estudio fue determinar el desarrollo posnatal de los Islotes de Langerhans (IL) en alpacas de 0 a 45 días de edad. Los animales fueron agrupados en nueve grupos etarios con rangos de cinco días se edad. El número de IL varió entre 3.03 a 4.46 en campos de 10x (con ocular de 10x), el diámetro de IL entre 67.89 a $72.55 \mu \mathrm{m}$, el número de células $\beta$ dentro del IL de 23.79 a 47.08 y el resto de células de 18.15 a 39.93 . Por otra parte, el receptor de insulina y la proteína de transporte GLUT-2 fueron observadas mediante la técnica de inmunohistoquímica a partir del primer día de edad. Se concluye que los IL incrementan en número y tamaño con la edad ( $\mathrm{p}<0.05)$, mientras que las células $\beta$ no muestran esta tendencia.

Palabras clave: páncreas endocrino, crías de alpaca, desarrollo neonatal

\section{Abstract}

The aim of this study was to determine the postnatal development of Islets of Langerhans (IL) in alpacas of 0 to 45 days of age. The animals were divided in nine age groups with range of five days. The mumber of IL varied from 3.03 to 4.46 in 10x fields (with an ocular of 10x), the IL diameter varied from 67.89 to $72.55 \mu \mathrm{m}$, the number of $\beta$ cells in the IL from 23.79 to 47.08 and other cells from 18.15 to 39.93. Moreover, the insulin

\footnotetext{
${ }^{1}$ Estación Experimental El Mantaro, Centro de Investigaciones IVITA, Universidad Nacional Mayor de San Marcos, Junín, Perú

${ }^{2}$ Laboratorio de Farmacología y Toxicología Veterinaria, ${ }^{3}$ Laboratorio de Fisiología Animal, Facultad de Medicina Veterinaria, Universidad Nacional Mayor de San Marcos, Lima, Perú

${ }^{4}$ Laboratorio de Patología, Servicio Nacional de Sanidad Agraria (SENASA), Lima, Perú

${ }^{5}$ E-mail: joserodriguezmv@gmail.com
}

Recibido: 5 de octubre de 2014

Aceptado para publicación: 25 de marzo de 2015 
receptor and the transport protein GLUT-2 were observed by immunohistochemistry on the first day of life. It is concluded that the number and size of IL increased with age $(\mathrm{p}<0.05)$ while $\beta$ cells do not show the same trend.

Key words: endocrine pancreas, baby alpaca, neonatal development

\section{INTRODUCCIÓN}

La glucosa es la principal fuente de energía que utiliza la célula. En los mamíferos, la regulación de la concentración del azúcar en sangre es esencial para el organismo y se realiza mediante un equilibrio entre el flujo de glucosa dentro y fuera del espacio extracelular, a través de la secreción coordinada de insulina y glucagón (Unger, 1991; DeFronzo, 1997).

La morfogénesis y la diferenciación del páncreas y sus tipos celulares son muy conservados entre los vertebrados durante el desarrollo fetal. El páncreas se desarrolla como estructura de yemas simples en el tubo del intestino primitivo a un órgano muy ramificado con muchos tipos de células especializadas (Kim y MacDonald, 2002). El páncreas maduro es un órgano bifuncional constituido principalmente por tejido exocrino organizado en acinos que segregan zimógenos con fines digestivos en la luz intestinal; en tanto que la otra porción pancreática, incorporado dentro del tejido exocrino, está conformada por los Islotes de Langerhans, que albergan los distintos tipos de células endocrinas (Slack, 1995).

Los islotes contienen un número variado de células, que mediante técnicas de inmunohistoquímica y procedimientos de tinción especializados, se pueden identificar tres tipos principales: células $\alpha, \beta$ y $\delta$, que son irregulares y se encuentran mezcladas y dispersas por todo el islote (Cabrera et al., 2006). Además, hay otros tipos de células en menor proporción, como las células de secreción de polipéptido pancreático (PP) y las células dendríticas. Las secreciones producto de estas células son: célula $\alpha$, glucagón; célula $\delta$, somatostatina y gastrina; célula dendrítica, antígeno clase II con capacidad fagocítica (Leprini et al., 1987); célula PP, polipéptido pancreático; y célula $\beta$, insulina y el polipéptido amiloide del islote (IAPP). Sin embargo, no todas las células â secretan IAPP (Iki y Pour, 2006).

El metabolismo de la glucosa en camélidos no es del todo explicado y son po$\cos$ los estudios relacionados a su metabolismo en crías de alpaca. Se sabe que las llamas y las alpacas mantienen altas concentraciones de glucosa en sangre (media: 7.0 $\mathrm{mmol} / \mathrm{L}$, rango: $4.6-8.9 \mathrm{mmol} / \mathrm{L}$ ) (Lassen $e t$ al., 1986; Fowler y Zinkl, 1989), más similar a la de un animal no rumiante; dado que estos últimos mantienen una baja concentración de glucosa en sangre (2.5-4.2 mmol/l) si se le compara con animales no rumiantes como el caballo (4.2-6.4 mmol/L) o el cerdo (4.7-8.3 $\mathrm{mmol} / \mathrm{L}$ ) (Kaneko, 1989). Las llamas y las alpacas muestran, además, una respuesta hiperglicémica extrema (concentraciones de glucosa en sangre entre 11.1 a $16.6 \mathrm{mmol} / \mathrm{L}$ ) en respuesta a situaciones de estrés, incluso mínimas (Fowler y Zinkl, 1989; Cebra et al., 2001a,b). La glucosa elevada en la sangre en llamas y alpacas puede ser de alguna forma explicada por estudios que muestran una respuesta de resistencia lenta y moderada a la insulina, algo similar a una condición de diabetes humana (Cebra et al., 2001a,b).

El conocimiento del metabolismo glucostático en crías de alpacas tiene que ser explicado a fin de que estos conocimientos puedan servir para entender si trastornos a este nivel intervienen en la alta incidencia de 
fisiopatologías entéricas en crías de alpaca. El objetivo del estudio fue, por lo tanto, determinar el desarrollo posnatal del páncreas endocrino de crías lactantes de alpaca mediante el número y diámetro de los Islotes de Langerhans y el número de células beta pancreáticas.

\section{Materiales y Métodos}

\section{Lugar de Ejecución}

El presente estudio se llevó a cabo en comunidades alpaqueras de los departamentos de Puno y Cusco, situadas por encima de los $3800 \mathrm{msnm}$. El procesamiento de las muestras se hizo en el Laboratorio de Fisiología Animal de la Facultad de Medicina Veterinaria de la Universidad Nacional Mayor de San Marcos y en el Laboratorio de Patología Animal del Servicio Nacional de Sanidad Agraria (SENASA), en el departamento de Lima, Perú.

\section{Animales y Muestras}

Se utilizaron 36 crías de alpacas de 0 a 45 días de edad. Los animales fueron divididos en nueve grupos etarios, cada uno formando un bloque de cinco días de edad. Se tomaron muestras de páncreas, se fijaron en formol al $15 \%$ por 48 horas o más y se redujeron a un tamaño aproximado de $0.5 \times 0.5 \times$ $0.5 \mathrm{~cm}$ para el procesamiento de parafinado.

Las muestras en parafina fueron seccionadas a un grosor de $4 \mu \mathrm{m}$ y teñidas con hematoxilina-eosina (H-E). Otro grupo fue seccionado a un grosor de $6 \mu \mathrm{m}$, donde la mitad fue teñido con Tricrómico de Gomori (para diferenciar células $\beta$ de otros tipos celulares del páncreas endocrino) y la otra parte fue procesada por inmunohistoquímica (IHQ), donde se usaron anticuerpos policlonales específicos contra el receptor de insulina (RI) y el transportador de glucosa 2 (GLUT2), siguiendo el protocolo del laboratorio fabricante (Santa Cruz Biotechnology, EEUU).

Se visualizaron los Islotes de Langerhans (IL) y las células $\beta$ (del resto celular) mediante el uso del microscopio Zeiss Primo Star, y con ayuda del software de medición bidimensional Axion Vision (Carl Zeiss, Alemania) se determinó el crecimiento de los islotes (en micras). Además, se determinó el número de islotes por campo de 10X (y con ocular de 10X) y el número celular por islote (Lis et al., 2003).

Los resultados se evaluaron mediante la prueba de análisis de varianza de una sola vía con un nivel de confianza del 95\%. Asimismo, se realizó la observación por IHQ del RI y GLUT 2 como proteínas que demuestran funcionalidad de las células â pancreáticas.

\section{Resultados}

El número de IL varió entre 3.03 a 4.46 (Fig. 1), el diámetro varió entre 67.89 a 72.55 $\mu \mathrm{m}$ (Fig. 2), y el número promedio de células $\beta$ dentro de los IL varió entre 23.79 a 47.08 y el resto de células entre 18.15 a 39.93 (Fig. $3)$. Las variaciones en frecuencia en relación al grupo etario 1 (0-5 días de edad) se muestran en el Cuadro 1. Asimismo, el receptor de insulina y la proteína de transporte GLUT-2 se apreciaron desde el primer día de edad de los animales.

Se puede observar que tanto el número como el diámetro de los IL han incrementado acorde con la edad de los animales, mostrando diferencias estadísticas entre los grupos etarios extremos. Así entre 1 y 3 versus 9 en el caso del número de IL (Fig. 1) y entre 1, 3 y 4 versus 9 para el diámetro de los IL (Fig. 2). 


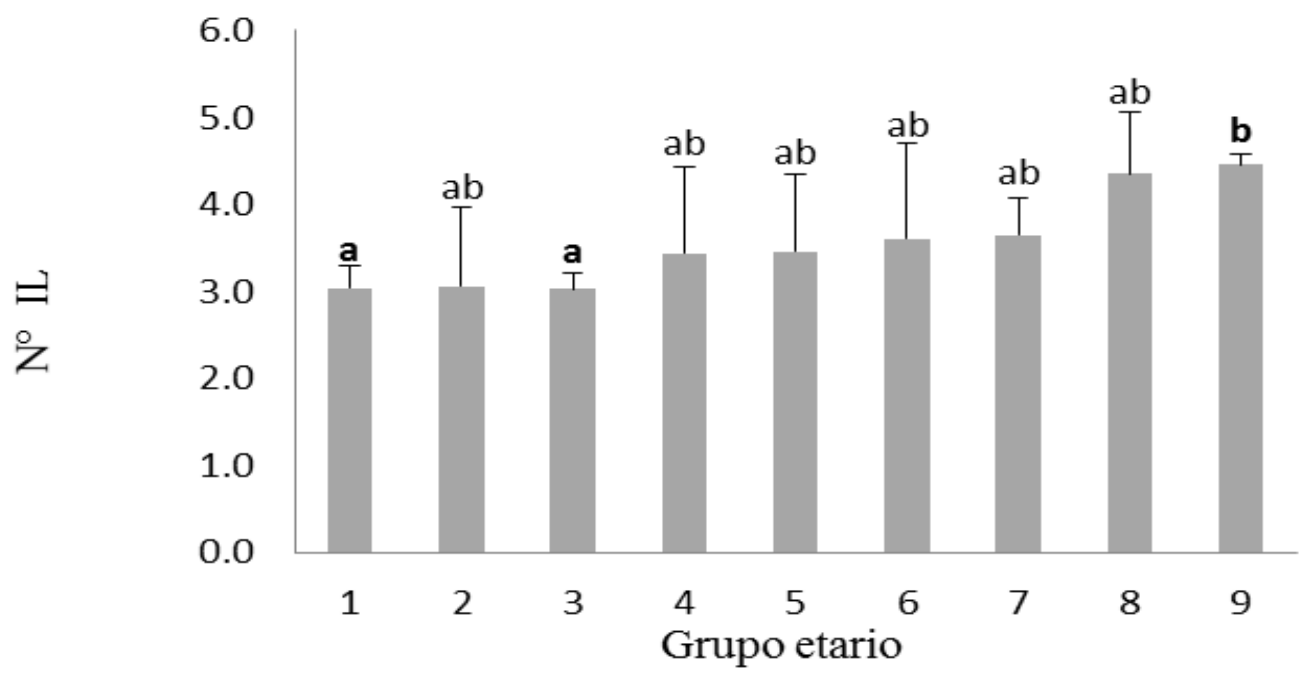

Figura 1. Número de los Islotes de Langerhans (IL) en tejido pancreático de nueve grupos etarios de alpacas ([1]: 0-5, [2]: 6-10, [3] 11-15, [4]: 16-20, [5]: 21-25, [6]: 26-30, [7]: 31-35, [8]: 36-40, [9]: 41-45 días de edad). Grupos con letras a y b son estadísticamente diferentes $(\mathrm{p}<0.05)$

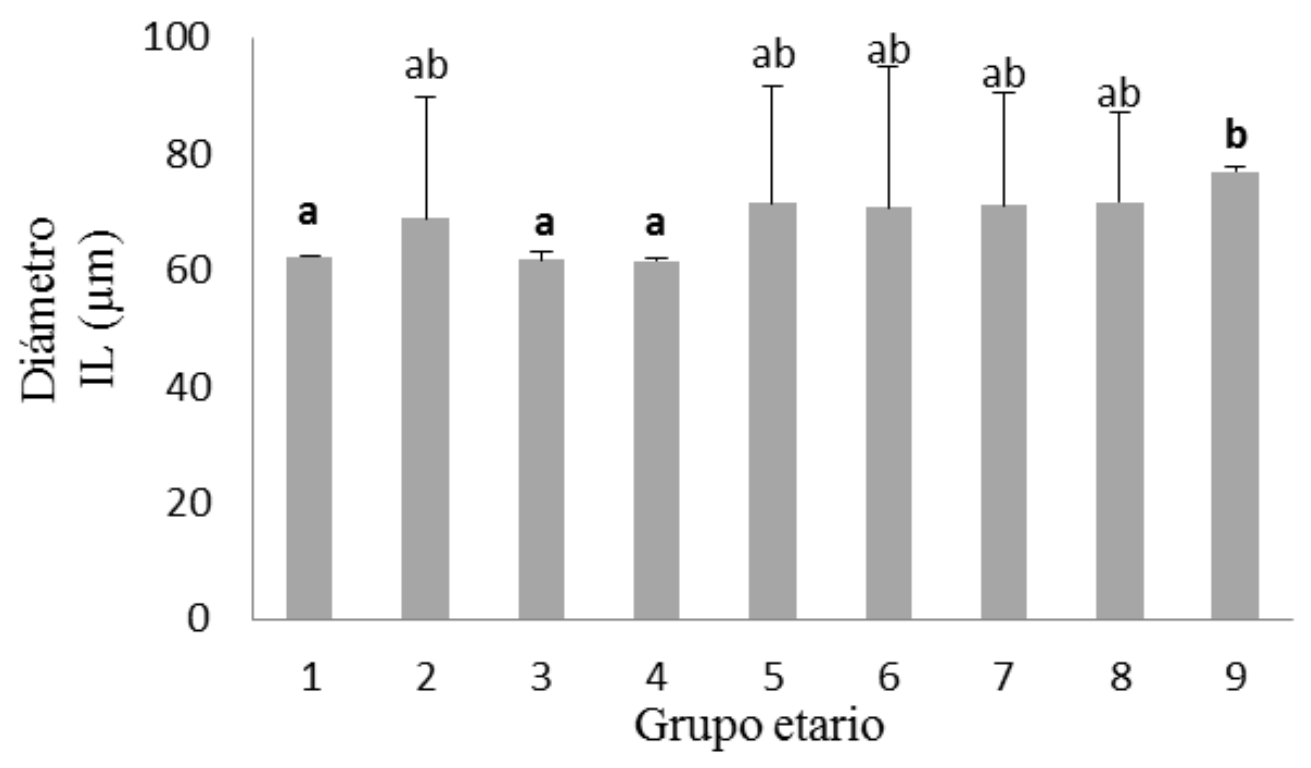

Figura 2. Diámetro de los Islotes de Langerhans (IL) en tejido pancreático de nueve grupos etarios de alpacas ([1]: 0-5, [2]: 6-10, [3] 11-15, [4]: 16-20, [5]: 21-25, [6]: 26-30, [7]: 31-35, [8]:36-40, [9]: 41-45 días de edad). Grupos con letras a y b son estadísticamente diferentes $(\mathrm{p}<0.05)$ 


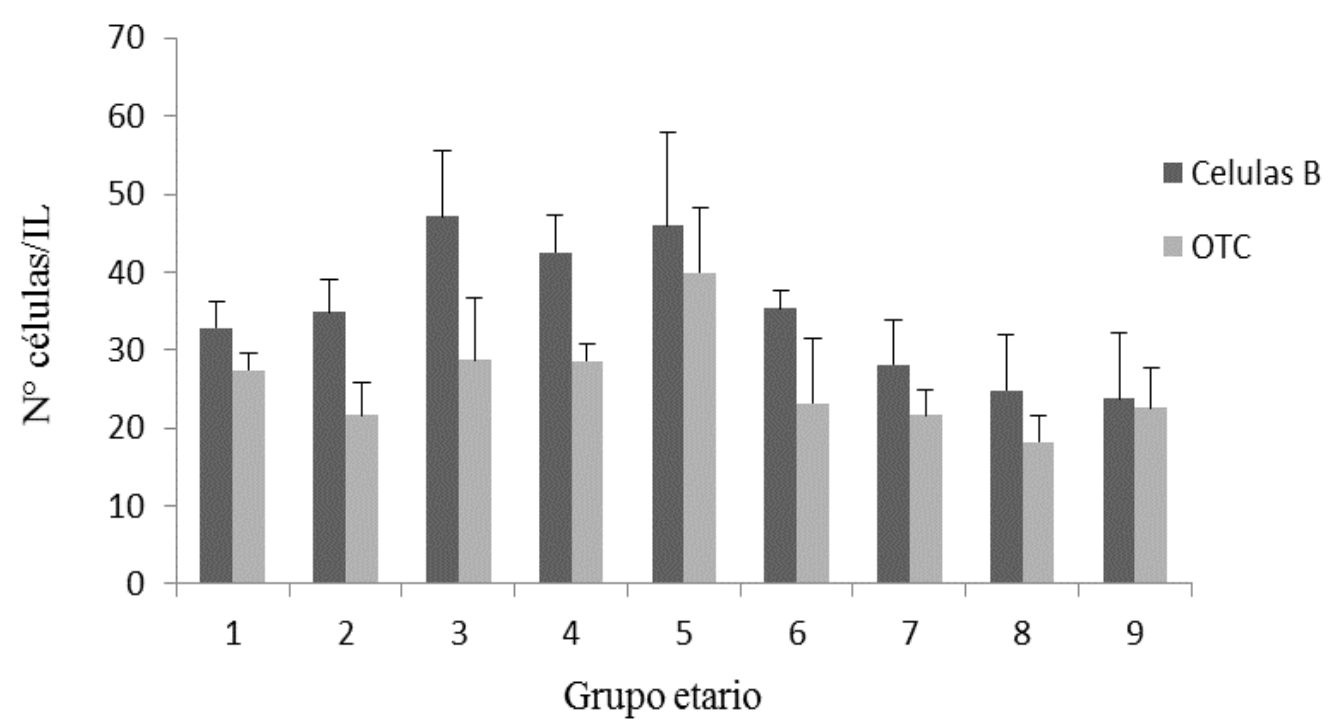

Figura 3. Número de células $\beta$ y otros tipos celulares (OTC) dentro de los Islotes de Langerhans (IL), en tejido pancreático de nueve grupos etarios de alpacas ([1]: 0-5, [2]: 6-10, [3] 11-15, [4]: 16-20, [5]: 21-25, [6]: 26-30, [7]:31-35, [8]:36-40, [9]: 41-45 días de edad)

Cuadro 1. Variaciones porcentuales ${ }^{1}$, acorde con la edad, de las características celulares de los Islotes de Langerhans (IL) en relación al grupo etario

\begin{tabular}{|c|c|c|c|c|c|c|c|c|c|}
\hline & \multicolumn{9}{|c|}{ Grupo etario $^{2}$} \\
\hline & 1 & 2 & 3 & 4 & 5 & 6 & 7 & 8 & 9 \\
\hline N. ${ }^{o}$ de IL & 1 & 1.00 & 1.00 & 1.13 & 1.14 & 1.18 & 1.20 & 1.43 & 1.47 \\
\hline Diámetro de IL & 1 & 1.02 & 1.00 & 1.01 & 1.05 & 1.04 & 1.05 & 1.06 & 1.07 \\
\hline Células beta & 1 & 1.06 & 1.43 & 1.29 & 1.40 & 1.07 & 0.86 & 0.75 & 0.72 \\
\hline Otros tipos celulares & 1 & 0.79 & 1.05 & 1.04 & 1.46 & 0.85 & 0.79 & 0.66 & 0.82 \\
\hline $\begin{array}{l}\text { Relación células beta / } \\
\text { total células }\end{array}$ & 0.6 & 0.6 & 0.6 & 0.6 & 0.5 & 0.6 & 0.6 & 0.6 & 0.5 \\
\hline
\end{tabular}

${ }^{1}$ Valores basados en el grupo etario 1

${ }^{2}$ [1]: 0-5, [2]: 6-10, [3] 11-15, [4]: 16-20, [5]: 21-25, [6]: 26-30, [7]: 31-35, [8]: 36-40, [9]: 41-45 días de edad

\section{Discusión}

La homeostasis glucémica se debe principalmente al correcto funcionamiento del páncreas endocrino, a través de sus secreciones hormonales como son la insulina y el glucagón. Alteraciones en este sistema endocrino causarán trastornos metabólicos hipo o hiperglucémicos (DeFronzo, 1997), de allí la necesidad de conocer el desarrollo posnatal de los IL para la interpretación de los cambios glucostáticos que ocurren en el animal. Diversos estudios en el humano sobre los mecanismos del IL posnatal y el crecimiento de las células $\beta$ han sido publicados 
(Bonner-Weir y Sharma, 2006; Granger y Kushner, 2009; Rankin y Kushner, 2009).

Los resultados encontrados guardan relación con otros estudios en mamíferos, como es el caso del cuy (Rodríguez et al., 2012), donde se evidenciaron diferencias significativas entre animales recién nacidos y destetados. En forma similar, en humanos se observa que la masa de células $\beta$ aumenta con la edad, presentando las mayores tasas de crecimiento durante los primeros seis años de vida (Meier et al., 2008). Por otro lado, en roedores (Finegood et al., 1995; Scaglia et al., 1997; Trudeau et al., 2000; Herbach et $a l ., 2005)$ se ha podido demostrar que la expansión temprana de los IL está asociada con una alta tasa de replicación celular dentro de los islotes ya existentes y no por la neogénesis a partir de precursores o células madre (Dor et al., 2004; Bouwens y Rooman, 2005; Nir et al., 2007; Dor y Melton, 2008).

El páncreas endocrino en la rata sufre una remodelación durante la segunda semana después del nacimiento. La masa de células $\beta$ no aumenta a pesar de una alta replicación de células $\beta$ pues a la vez se presenta un alto nivel de apoptosis (Scaglia et al., 1997). Este fenómeno probablemente sucedería en el páncreas endocrino de alpacas neonatas, donde se muestra que hay una disminución del número de células $\beta$ pancreáticas en algunos grupos etarios (Cuadro 1).

En el estudio de Rodríguez et al. (2012) en cuyes lactantes, se determinó que las células $\beta$ fueron observadas desde el primer día de edad, de forma similar al presente estudio. Sin embargo, el número de células $\beta$ de los IL en el páncreas endocrino de cuyes se incrementaron acorde con la edad, a diferencia del presente estudio en alpacas. Además, el aumento celular en cuyes presentó mesetas, en forma similar a observaciones en la rata (Svenstrup et al., 2002). Por otro lado, en el cerdo, el volumen promedio de las células $\beta$ aumenta progresivamente en el periodo posnatal, llegando a una meseta a los 60 días de edad (Bock et al., 2003).
Según el Cuadro 1, el número de células $\beta$ en la alpaca no presenta un cambio significativo con la edad, tal vez debido a que el tejido del islote se 'diluye' por el crecimiento posnatal más exuberante del tejido exocrino después del nacimiento. Sin embargo, nuevas células surgen por neogénesis o diferenciación de las células endocrinas del islote del epitelio ductal o por la replicación de las células de islotes existentes (Hellerstrom $e t$ al., 1988; Finegood et al., 1995; Montanya et al., 2000).

La identificación de células $\beta$ desde el primer día de edad en alpacas al igual que en cuyes (Rodríguez et al., 2012), mediante la tinción específica de Tricrómico de Gomori, indica que el proceso de diferenciación celular en el páncreas endocrino comienza en la etapa fetal. Este proceso de diferenciación celular se debe a la proteína PDX1, la cual genera la maduración de las células de los islotes (Jonsson et al., 1994; Harrison et al., 1999), la diferenciación de las células $\beta$ (Kojima et al., 2002; Imai et al., 2005), así como el mantenimiento de la madurez y función de las células $\beta$ mediante la regulación de varios genes relacionados con estas células (Holland et al., 2005).

Otro consideración en este estudio es que las células $\beta$ representaron más del 50\% de las células totales dentro del islote, lo que guarda relación con el número de células $\beta$ en cuyes $(65 \%$ del total de células dentro de los islotes en todas las edades) (Rodríguez et al., 2012).

\section{Conclusiones}

- El número y tamaño de los Islotes de Langerhans en la alpaca incrementan con la edad neonatal.

- Las células dentro del IL, con las técnicas empleadas, no muestran un crecimiento significativo en tamaño con la edad del animal. 


\section{Literatura Citada}

1. Bock T, Kyhnel A, Pakkenberg B, Buschard K. 2003. The postnatal growth of the beta-cell mass in pigs. $\mathbf{J}$ Endocrinol 179: 245-252.

2. Bonner-Weir S, Sharma A. 2006. Are there pancreatic progenitor cells from which new islets form after birth? Nat Clin Pract Endocrinol Metab 2: 240-241. doi: $10.1038 /$ ncpendmet0186

3. Bouwens L, Rooman I. 2005. Regulation of pancreatic beta-cell mass. Physiol Rev 85: 1255-1270. doi: 10.1152/ physrev.00025.2004

4. Cabrera O, Berman D, Kenyon N, Ricordi C, Berggren P, Caicedo A. 2006. The unique cytoarchitecture of human pancreatic islets has implications for islet function. Proc Natl Acad Sci USA 103: 2334-2339. doi: 10.1073/ pnas.0510790103

5. Cebra C, McKane S, Tornquist S. 2001a. Effects of exogenous insulin on glucose tolerance in alpacas. Am J Vet Res 62: 1544-1547.

6. Cebra C, Tornquis S, Van Saun R, Smith B. 2001b. Glucose tolerance testing in llamas and alpacas. Am J Vet Res 62: 682-686. doi: 10.2460/ ajvr.2001.62.682

7. DeFronzo R. 1997. Pathogenesis of type 2 diabetes, metabolic and molecular implications for identifying diabetes genes. Diab Rev 5: 177-269.

8. Dor Y, Brown J, Martinez OI, Melton DA. 2004. Adult pancreatic beta-cells are formed by self-duplication rather than stem-cell differentiation. Nature 429: 4146. doi: $10.1038 /$ nature 02520

9. Dor Y, Melton DA. 2008. Facultative endocrine progenitor cells in the adult pancreas. Cell 132: 183-184. doi: 10.1016/ j.cell.2008.01.004.

10. Finegood D, Scaglia L, Bonner-Weir $S$. 1995. Dynamics of $\beta$-cell mass in the growing rat pancreas: estimation with a simple mathematical model. Diabetes 44: 249-256. doi: 10.2337/diab.44.3.249
11. Fowler M, Zinkl J. 1989. Reference ranges for hematologic and serum biochemical values in llamas. Am J Vet Res 50: 2049-2053.

12. Granger A, Kushner JA. 2009. Cellular origins of beta-cell regeneration: a legacy view of historical controversies. J Intern Med 266: 325-338. doi: 10.1111/j.13652796.2009.02156.x

13. Harrison K, Thaler J, Pfaff S, Gu H, Kehrl J. 1999. Pancreas dorsal lobe agenesis and abnormal islets of Langerhans in Hlxb9-deficient mice. Nat Genet 23: 71-75.

14. Hellerstrom C, Swenne I, Andersson A. 1988. Islet cell replication and diabetes. In: Lefebvre PJ, Pipeleers DG (eds). The pathology of the endocrine pancreas in diabetes. Heidelberg, Germany: Springer-Verlag. $141 \mathrm{p}$.

15. Herbach N, Göke B, Schneider M, Hermanns W, Wolf E, Wanke R. 2005. Overexpression of a dominant negative GIP receptor in transgenic mice results in disturbed postnatal pancreatic islet and beta-cell development. Regul Pept 125: 103-117. doi: 10.1016/j.regpep.2004. 08.021

16. Holland A, Hale M, Kagami H, Hammer R, Macdonald R. 2005. Experimental control of pancreatic development and maintenance. Proc Natl Acad Sci USA 99: 12236-12241. doi : 10.1073/pnas.192255099

17. Iki K, Pour P. 2006. Distribution of pancreatic endocrine cells, including IAPP-expressing cells in non-diabetic and type 2 diabetic cases. J Histochem Cytochem 55: 111-118.

18. Imai J, Katagiri H, Yamada T, Ishigaki Y, Ogihara T, Uno K, Hasegawa Y, et al. 2005. Constitutively active PDX1 induced efficient insulin production in adult murine liver. Biochem Biophys Res Commun 326: 402-409. doi:10.1016/j.bbrc.2004.11.047

19. Jonsson J, Carlsson L, Edlund T, Edlund H. 1994. Insulin-promoter-factor 1 is required for pancreas development in mice. Nature 371: 606-609. doi: $10.1038 / 371606 a 0$ 
20. Kaneko J. 1989. Clinical biochemistry of domestic animals. 4th ed. San Diego, USA: Academic Press. 932 p.

21. Kim S, MacDonald R. 2002. Signaling and transcriptional control of pancreatic organogenesis. Curr Opin Genet Dev 12: 540-547. doi: 10.1016/S0959-437X(02) 00338-6

22. Kojima H, Nakamura T, Fujita Y, Kishi A, Fujimiya M, Yamada S, Kudo $M$, et al. 2002. Combined expression of pancreatic duodenal homeobox 1 and islet factor 1 induces immature enterocytes to produce insulin. Diabetes 51: 13981408. doi: 10.2337/diabetes.51.5.1398

23. Lassen E, Pearson E, Long $P$, Schmotzer W, Kaneps A, Riebold T. 1986. Clinical biochemical values of llamas: reference values. Am J Vet Res 47:2278-2280.

24. Leprini A, Valente U, Celada F, Fontana I, Barocci S, Nocera A. 1987. Morphology, cytochemical features and membrane phenotype of HLA-DR+ interstitial cells in the human pancreas. Pancreas 2: 127-135.

25. Lis A, Barra F, Beltramino F, Peralta J, Rejf P. 2003. Páncreas: diferencias microestructurales y de indicadores funcionales. Sitio Argentino de Producción Animal. [Internet]. Disponible en: http:// www.produccion-animal.com.ar/ informacion_tecnica/destete/58 pancreas.pdf

26. Meier JJ, Butler AE, Saisho Y, Monchamp T, Galasso R, Bhushan A, Rizza R, Butler P. 2008. Beta-cell replication is the primary mechanism subserving the postnatal expansion of beta-cell mass in humans. Diabetes 57: 1584-1594. doi: 10.2337/db07-1369

27. Montanya E, Nacher V, Biarnés M, J Soler. 2000. Linear correlation between beta-cell mass and body weight throughout the lifespan in Lewis rats: role of beta-cell hyperplasia and hypertrophy. Diabetes 49: 1341-1346. doi: 10.2337/diabetes.49.8.1341

28. Nir T, Melton DA, Dor Y. 2007. Recovery from diabetes in mice by beta cell regeneration. J Clin Invest 117: 25532561. doi: 10.1172/JCI32959

29. Rankin MM, Kushner JA. 2009. Adaptive beta-cell proliferation is severely restricted with advanced age. Diabetes 58: 1365-1372. doi: 10.2337/ $\mathrm{db} 08-1198$

30. Rodríguez J, Cueva S, Vásquez, M, Lira B, Olivera L, Espinoza J. 2012. Desarrollo postnatal del páncreas endocrino de cuyes (Cavia porcellus) lactantes. Rev Inv Vet Perú 23: 13-19. doi: 10.15381/rivep.v23i1.877

31. Scaglia L, Cahill C, Finegood D, Bonner-Weir S. 1997. Apoptosis participates in the remodeling of the endocrine pancreas in the neonatal rat. Endocrinology 138:1736-1741. doi : 10.1210/endo.138.4.5069

32. Slack J. 1995. Developmental biology of the pancreas. Development 121: 15691580.

33. Svenstrup K, Skau M, Pakkenberg B, Buschard K, Bock T. 2002. Postnatal development of beta-cells in rats. Proposed explanatory model. Acta Patholog Microb 110: 372-378.

34. Trudeau JD, Dutz JP, Arany E, Hill DJ, Fieldus WE, Finegood DT. 2000. Neonatal beta-cell apoptosis: a trigger for autoimmune diabetes? Diabetes 49: 1-7. doi: 10.2337/diabetes.49.1.1

35. Unger R. 1991. Diabetic hyperglycemia: link to impaired glucose transport in pancreatic beta cells. Science 251: 12001205. doi: 10.1126/science. 2006409 